But other geneticists say that intelligence is so complex a trait, influenced by so many genes, that not even a sample of thousands of people would yield sufficient statistical power to get at its genetic basis. They point to studies such as one published in May that examined the genomes of more than 125,000 people and found only three genetic markers with a small effect on how long an individual stays at school (C. A. Rietveld et al. Science 340, 1467-1471; 2013). Project Einstein "is unlikely to have any statistical power", says geneticist Daniel MacArthur at Massachusetts General Hospital in Boston who, with colleagues, has amassed a pedigree of 13 million related people to try to tackle the heritability of complex traits (see Nature http://doi.org/ppj; 2013).

Some participants in Project Einstein are intrigued by the chance to learn about their own genetic sequences, which will be shared with them. "As a science-fiction fan, I like the idea of having my own genome sequenced," says David Aldous, a mathematician at the University of California, Berkeley. "Maybe I'll print a segment onto a T-shirt."

Others say they wouldn't be surprised if the study found that maths aptitude was not born so much as made. "I feel that the notion of 'talent' may be overrated," says Michael Hutchings, a mathematician also at Berkeley. He adds that even if genetic markers are found, they could be used for good - not to pre-select for maths geniuses, but to help parents to understand the particular abilities of their children and give them the support they need.

However, McMullen is concerned that the project is appealing to participants' self-interest without disclosing enough information. "I thought it was strange that it was called 'Project Einstein', which seemed designed to appeal to the participants' egos," he says. He asked the project's staff and the New England Institutional Review Board, which approved the study, to explain how results would be used. "The uniform answer to my questions was that 'we are not responsible for how the information is used after the study is completed"' he says.

The Rothberg Institute for Childhood Diseases, Rothberg's private foundation based in Guilford, Connecticut, is the study's sponsor. But Rothberg won't say who is funding the project, which other geneticists estimate will cost at least US\$1 million. Some speculate that Rothberg is funding it himself. In 2001, Fortune estimated his net worth to be $\$ 168$ million, and that was before he sold the sequencing companies he founded - 454 Life Sciences and Ion Torrent, both based in Connecticut - for a combined total of $\$ 880$ million.

Rothberg is adamant that the project is well worth the time and the money, whoever is paying for it. "This study may not work at all," he says - before adding, quickly, that it "is not a crazy thing to do". For a multimillionaire with time on his hands, that seems to be justification enough.

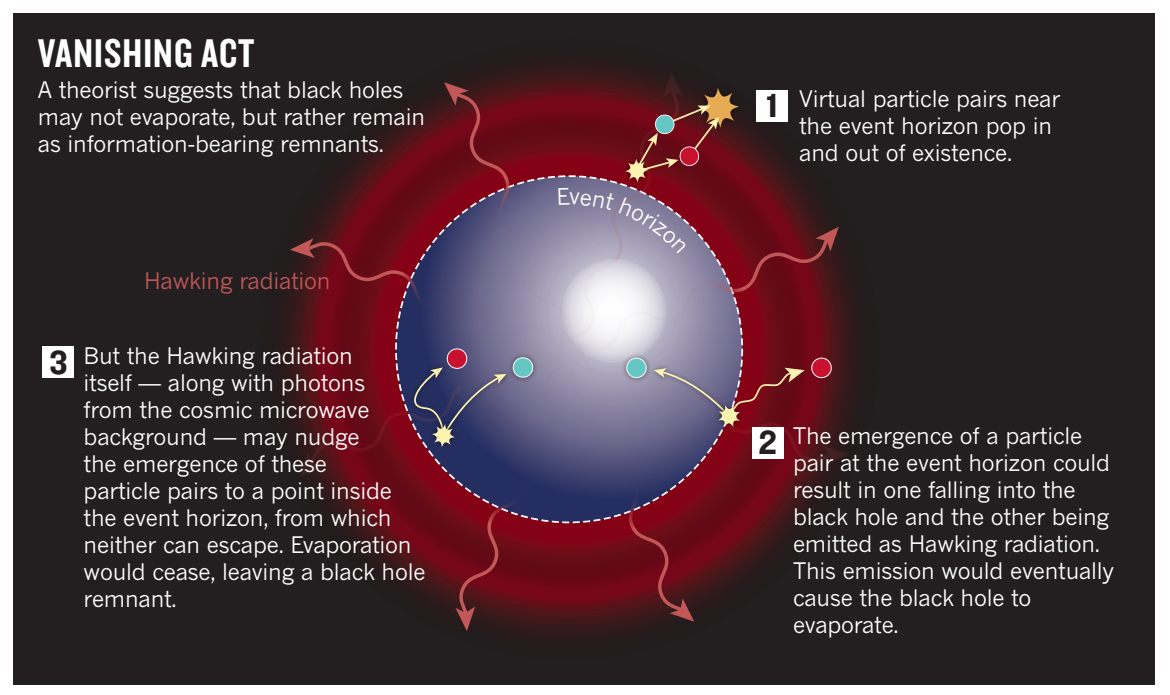

ASTROPHYSICS

\title{
Black holes shrink but endure
}

\section{Theorist's idea takes on information-preservation problem.}

\section{BY RON COWEN}

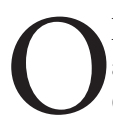
ld black holes never die, they just fade away. So says veteran cosmologist George Ellis of the University of Cape Town in South Africa, who suggests that the cosmos may be littered with an untold number of shrunken black hole remnants.

Ellis' speculative report, posted on 17 October on the preprint server $\operatorname{arXiv}$ (G. F. R. Ellis http://arxiv.org/abs/1310.4771;2013), seems to undermine the seminal work of Stephen Hawking, a cosmologist at the University of Cambridge, UK. In 1974, Hawking calculated that, owing to quantum effects, black holes are not entirely black: some particles escape the black hole's gravitational barrier, known as the event horizon. For a solar-mass black hole, these particles, known as Hawking radiation, would be emitted over the course of $10^{67}$ years until the object vanished without a trace (S. W. Hawking Nature 248, 30-31; 1974).

Although many physicists are sceptical about Ellis's work, it highlights a long-running debate over the ultimate fate of black holes. Uncertainties abound because of the difficulties in reconciling quantum theory which predicts the Hawking radiation - and Einstein's classical theory of gravitation, which defines a black hole's structure. "This is very much a living issue that people are confused about," says Vijay Balasubramanian, a string theorist at the University of
Pennsylvania in Philadelphia.

The debate also touches on one of the most cherished beliefs about the Universe: that information is always preserved. If black holes evaporate, then the information they contain may die along with them. By contrast, a black hole remnant would offer a way in which information might be preserved (even if it could never be extracted). By serving as a storehouse, a remnant "could be a fantastic way of resolving all the issues we have with black holes", says theoretical physicist Jeff Murugan of the University of Cape Town.

In Hawking's original view, quantum theory permits large fluctuations in energy for brief moments of time. As a consequence, the vacuum of space seethes with particle-antiparticle pairs that continually pop in and out of existence (see 'Vanishing act'). When this occurs at the event horizon, one member of a particle pair could be sucked into the black hole, whereas the other could escape as Hawking radiation, depleting the black hole's mass.

But Ellis says that this radiation has another effect. According to Einstein, any source of mass or energy distorts space. A black hole, a body so massive that space closes in on itself, is an extreme example of that distortion. The Hawking radiation would add even more distortion, Ellis says, and so, too, would the ubiquitous photons from the cosmic microwave background, the bath of radiation left over from the Big Bang. He says that these two 
types of radiation bend space-time in such a way that the region from which the Hawking radiation is generated eventually gets pushed back behind the event horizon. Once it has been relocated, the radiation can no longer escape and the black hole plateaus at a constant mass.

The analysis is more of an essay than a fullblown study, says string theorist Samir Mathur of Ohio State University in Columbus, because Ellis does not perform a thorough calculation for the bending effect of the radiation. Other physicists say that Ellis is probably incorrect. Nonetheless, Balasubramanian says, the paper is an example of physicists' innate dissatisfaction with evaporating black holes. "The stuff radiates in a weird way, information is lost and then we turn intellectual somersaults to try to account for why the information isn't lost," he says.

But black hole remnants do not offer a perfect solution to the problem of information loss, either. To contain all the information originally stored in a large black hole, the tiny remnants would need to have an infinite number of internal states - which would violate quantum theory, says Mathur.

In 1997, Mathur found a potential way around this problem. He and his colleagues used string theory, a way of marrying quantum theory and gravity, to describe all of the possible states of the gravitational field of black holes. They found that these states lay just outside the classical event horizon in a 'fuzzball' of quantum states. If the fuzzball was the black hole's true boundary, then there would be no Hawking radiation emitted from the event horizon, no evaporation and no information loss, he says.

Last year, others proposed a more violent approach to the problem of information loss. They suggested that the particles in the Hawking radiation did not behave randomly but were instead 'entangled' with each other in such a way that they could be messengers from the darkness, conveying information that had been stored in the black hole. But that entanglement had its own consequence: an enormous amount of energy would be unleashed at the event horizon, such that anything falling in would be instantly immolated (see Nature 496, 20-23; 2013).

The ongoing struggle to preserve the precepts of quantum theory without losing information may offer clues about how and where quantum physics begins to significantly modify Einstein's theory of gravity, says Matt Visser, a cosmologist at Victoria University of Wellington. The conventional picture holds that quantum theory makes big corrections to gravity only well inside the event horizon, near the black hole's singularity - the point at which the density of matter becomes infinite. But some physicists think that quantum physics may be blurring the sharp boundary of the event horizon itself. Ellis's work, Visser says, puts a stronger spotlight on such speculations. -

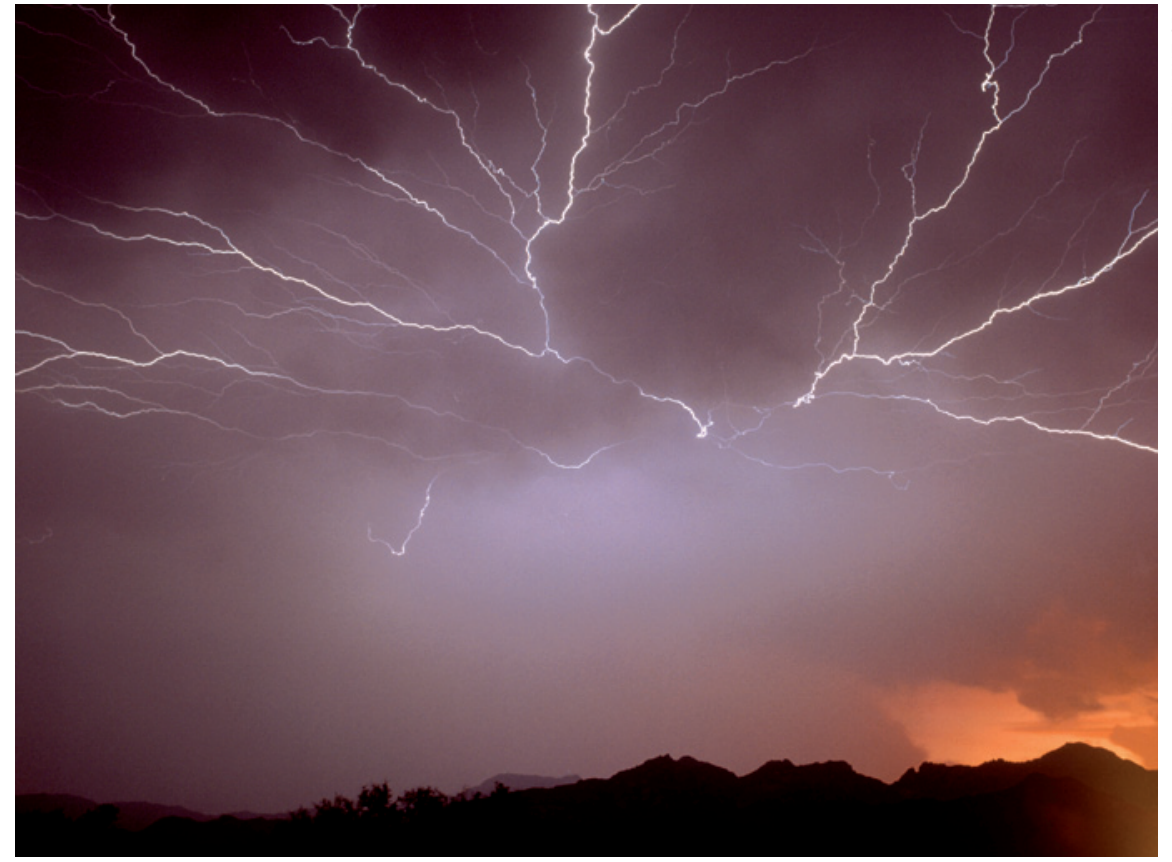

Cloud-to-cloud lightning can be detected with sensors, and used as a proxy for intense storms.

\title{
METEOROLOGY
}

\section{Lightning network tested out in Guinea}

\author{
Project to forecast African storms provides cheap \\ alternative to radar-based weather services.
}

\section{BY JEFF TOLLEFSON}

$\mathrm{M}$ eteorologists watched as afternoon thunderstorms brewed in the mountainous region of central Guinea. By the evening of 22 October, the storms had intensified and were moving west towards the coast of Africa. At 8.20 p.m., the meteorologists received a thunderstorm alert, and for the next 45 minutes the 130,000 residents of the city of Fria were hammered by heavy rain, flash floods and winds of up to 77 kilometres per hour.

What happened that evening was not unusual. Similar storms blow through Fria and Guinea's coastal capital Conakry regularly during the rainy season. Flash flooding is a common problem, and the country is frequently buffeted by tornadoes.

What was unusual was the way the storm was detected. Government meteorologists in Guinea lack the Doppler radar system that is usually used for this, and have struggled to track weather using rudimentary equipment. Europe and the United States provide free satellite data and forecasts, but these are coarse and infrequent. Only in recent months has Guinea turned to a new, simple proxy for storms: flashes of lightning.

Lightning-detection sensors installed atop just 12 mobile-phone towers now allow the country's meteorological service to track storms nationwide as they develop (see 'Lightning fast'). "With this project, we receive virtually real-time data throughout the entire country," says Mamadou Lamine Bah, director of the service.

The project shows how lightning detection could provide a quick and relatively cheap way for poor countries to acquire basic weather services. Earth Networks, based in Germantown, Maryland, spent around US\$1 million to deploy the current network. By comparison, Bah says, even a simple Doppler radar system would cost around \$10 million. And beyond that initial outlay, a lack of expertise in countries such as Guinea makes those systems prohibitively difficult to maintain and operate.

Earth Networks is currently giving Guinea its lightning data for free, but eventually hopes to sell its services there. Already, the company 